\title{
Microstructural Organization of the Cingulum Tract and the Level of Default Mode Functional Connectivity
}

\author{
Martijn van den Heuvel, René Mandl, Judith Luigjes, and Hilleke Hulshoff Pol \\ Rudolf Magnus Institute of Neuroscience, University Medical Center Utrecht, 3508 GA Utrecht, The Netherlands
}

The default mode network is a functionally connected network of brain regions that show highly synchronized intrinsic neuronal activation during rest. However, less is known about the structural connections of this network, which could play an important role in the observed functional connectivity patterns. In this study, we examined the microstructural organization of the cingulum tract in relation to the level of resting-state default mode functional synchronization. Resting-state functional magnetic resonance imaging and diffusion tensor imaging data of 45 healthy subjects were acquired on a 3 tesla scanner. Both structural and functional connectivity of the default mode network were examined. In all subjects, the cingulum tract was identified from the total collection of reconstructed tracts to interconnect the precuneus/posterior cingulate cortex and medial frontal cortex, key regions of the default mode network. A significant positive correlation was found between the average fractional anisotropy value of the cingulum tract and the level of functional connectivity between the precuneus/posterior cingulate cortex and medial frontal cortex. Our results suggest a direct relationship between the structural and functional connectivity measures of the default mode network and contribute to the understanding of default mode network connectivity.

Key words: resting-state; functional connectivity; structural connectivity; default mode network; DTI; fMRI

\section{Introduction}

Multiple cortical and subcortical regions show coherent time series during rest and are suggested to form resting-state networks (RSNs) (Damoiseaux et al., 2006; van den Heuvel et al., 2008). In particular, a set of regions called the default mode network (Raichle et al., 2001; Greicius et al., 2003) has been reported to show high levels of intrinsic neuronal activity during rest (Gusnard et al., 2001; Greicius et al., 2003; Raichle and Snyder, 2007). The most often reported and most influential regions of this network are the precuneus/posterior cingulate cortex (PCC), involved in responding to salient stimuli in the world around us (Corbetta et al., 2000; Gusnard et al., 2001) and medial frontal cortex (MFC), involved in emotional processing and the monitoring of one's mental state (Northoff and Bermpohl, 2004; Northoff et al., 2006; Schmitz and Johnson, 2007). Neuronal activation patterns of the PCC and MFC have been reported to be highly coherent during rest (Greicius et al., 2003; Fox and Raichle, 2007), indicating a high level of functional connectivity (Biswal et al., 1995) and ongoing communication between the PCC and MFC during rest. This supports the idea of the default mode network as a cohesive brain network that may be involved in important functions of human cognition (Gusnard et al., 2001; Greicius et al., 2003; Mason et al., 2007).

\footnotetext{
Received June 27, 2008; revised July 30, 2008; accepted Aug. 27, 2008.

This work was supported by Dutch Science Organization for Medical Research (Nederlandse Organisatie voor Wetenschappelijk Onderzoek) ZON-MW VIDI Program Grant 917.46 .370 (H.H.P.).

Correspondence should be addressed to Martijn van den Heuvel, Rudolf Magnus Institute of Neuroscience, University Medical Center Utrecht, Heidelberglaan 100, P.0. Box 85500, 3508 GA Utrecht, The Netherlands. E-mail: m.p.vandenheuvel@umcutrecht.nl.

DOI:10.1523/JNEUROSCI.2964-08.2008

Copyright $\odot 2008$ Society for Neuroscience $\quad$ 0270-6474/08/2810844-08\$15.00/0
}

However, less is known about the structural connections of the default mode network. The cingulum bundle is a collection of white matter tracts that connect regions of the frontal lobe with the precuneus, posterior cingulate cortex, hippocampus, and parahippocampus (Wakana et al., 2004; Schmahmann et al., 2007; Lawes et al., 2008). These termination regions show large overlap with key regions of the default mode network. Indeed, Greicius et al. (2008) recently reported that the PCC and MFC regions of the default mode network are connected by the cingulum tract. These results suggest an anatomical basis of the default mode network and raise the question about a possible association between the structural and functional connectivity measures of the default mode network. We hypothesize that an association exists between the microstructural organization of the cingulum tract and the level of default mode functional connectivity. Examining this association could add to the understanding of default mode network connectivity. In this study, resting-state functional magnetic resonance imaging (fMRI) recordings and diffusion tensor imaging (DTI) scans were acquired on a 3 tesla MR scanner in 45 subjects. The unique level of functional connectivity between the PCC and MFC regions of the default mode network was computed as the partial correlation between their resting-state time series. Cingulum tracts interconnecting the PCC and MFC were reconstructed using the DTI scans, and fractional anisotropy (FA) values of these tracts were calculated as an indication of white matter integrity (Kim et al., 2007). Over the group of subjects, the mean FA value of the cingulum tract was correlated with the level of functional connectivity between the PCC and MFC regions of the default mode network.

These data have been published previously in abstract form (van den Heuvel et al., 2007). 


\section{Materials and Methods}

Subjects. Forty-five healthy subjects (mean \pm SD age, $24.8 \pm 4.8$ years; 25 male, 20 female) participated in this study after giving written consent as approved by the medical ethics committee for research in humans of the University Medical Centre Utrecht, The Netherlands. All subjects underwent a 45 min scanning session. During the resting-state recordings, subjects were instructed to relax, keep their eyes closed without falling asleep, and to think of nothing in particular.

Image acquisition. Resting-state $\mathrm{PMRI}$ and DTI data were acquired on a 3 tesla Philips Achieva Medical Scanner (Philips Medical Systems) at the University Medical Center Utrecht, The Netherlands. During the rest experiment, resting-state blood oxygenation level-dependent (BOLD) signals were recorded during a period of $8 \mathrm{~min}$ using a fast $\mathrm{fMRI}$ sequence [three-dimensional (3D) PRESTOSENSE p/s-reduction 2/2 (Golay et al., 2000; Neggers et al., 2008); repetition time (TR)/echo time (TE), 22 $\mathrm{ms} / 32 \mathrm{~ms}$ using shifted echo; flip angle, $9^{\circ}$; dynamic scan time, $0.5 \mathrm{~s} ; 1000$ timeframes; field of view (FOV), $256 \times 256 \mathrm{~mm} ; 4 \mathrm{~mm}$ isotropic voxel size; 32 slice volume covering the whole brain]. Directly after the fMRI time series, an additional functional scan was acquired with identical parameters but with a high anatomical contrast attributable to an increased flip angle of $25^{\circ}$. This additional high-contrast functional scan was acquired to improve the coregistration of the functional images with the anatomical image. In the same scanning session, DTI scans were acquired [DTI-MR using parallel imaging SENSE p-reduction 3; high angular gradient set of 30 weighted directions (Jones et al., 1999; Jones, 2004); TR, $7035 \mathrm{~ms}$; TE, $68 \mathrm{~ms}$; echo planar imaging (EPI) factor, 35; FOV, $240 \times 240 \mathrm{~mm} ; 2 \mathrm{~mm}$ isotropic voxel size; 75 slices covering the whole brain]. In total, two DTI sets of 30 weighted diffusion scans with different weighting directions $\left(b=1000 \mathrm{~s} / \mathrm{mm}^{2}\right)$ and two unweighted B0 scans $\left(b=0 \mathrm{~s} / \mathrm{mm}^{2}\right)$ were acquired. The second DTI set was acquired with a reversed $k$-space readout direction (anterior direction) compared with the first set (posterior direction) (Andersson et al., 2003). In addition, a T1-weighted image (3D fast field echo using parallel imaging; TR, $10 \mathrm{~ms}$; TE, $4.6 \mathrm{~ms}$; FOV, $240 \times 240 \mathrm{~mm}$; 200 slices; $0.75 \mathrm{~mm}$ isotropic voxel size) was acquired for anatomical reference of the functional time series and structural DTI scans.

Image preprocessing. fMRI preprocessing was performed with the SPM2 software package (http://www.fil.ion.ucl.ac.uk). The functional scans were corrected for small head movements by realigning all scans to the last functional scan. Both the T1 image and the functional scans were coregistered with the high-contrast functional scan to enable spatial overlap between the functional time series and the T1 image. Cortical voxels were selected based on a cortical segmentation of the T1 image. Cortical segmentation was performed with the widely used and freely available Freesurfer software package (http://surfer.nmr.mgh.harvard.edu/). The T1 image was normalized to match the Montreal Neurological Institute 305 T1 template brain (Collins et al., 1994). Next, the fMRI time series and cortical segmentation map were normalized to standard space by using the normalization parameters of the $\mathrm{T} 1$ image. The normalized cortical segmentation map was resliced to the spatial resolution of the fMRI images.

DTI preprocessing was performed with the diffusion toolbox of Andersson and Skare (2002) and in-house developed software. First, susceptibility distortions, often reported in single-shot EPI images, were corrected by combining the two sets of DTI images. A field distortion map was computed based on the two unweighted B0 images and applied to the weighted images (Andersson et al., 2003), resulting in a single set of corrected scans, which were realigned with the corrected B0 image (Andersson and Skare, 2002). For each voxel, the main diffusion direction was computed by fitting a tensor to the diffusion scans using a robust tensor fit method based on an M-estimator (Chang et al., 2005). The "fiber assignment by continuous tracking" (FACT) algorithm (Mori et al., 1999; Mori and van Zijl, 2002) was used to reconstruct the white matter tracts of the brain. In each voxel, 27 fiber seeds were started. Fiber tracking was stopped when the fiber touched a voxel with an FA value $<0.1$ or when it had an average angle change between the neighboring eigenvectors of $>45^{\circ}$ or when the trajectory of the traced fiber exceeded the brain. Only fibers with a minimum length of $50 \mathrm{~mm}$ were considered.
Next, the B0 image was registered (linear) to the anatomical image and normalized using the normalization parameters of the $\mathrm{T} 1$ image, to overlap with the normalized resting-state fMRI time series.

Selection of default mode network. RSNs across the group of subjects were selected with the voxel-based "normalized cut group clustering" approach, described in detail previously (van den Heuvel et al., 2008). This method involves the clustering of voxels that consistently show a high level of functional connectivity over a group of subjects. In brief, for each individual dataset, the resting-state fMRI dataset was represented as a network that was constructed out of all cortical voxels with weighted connections between all voxel pairs. Cortical voxels were identified from the individual cortical segmentation map. The weights of the connections between the voxels in the network were computed as the zero-lag temporal correlation between the filtered resting-state time series. The resulting connectivity graph was then clustered, resulting in the grouping of voxels that showed a high level of functional connectivity. Next, the overlap of the individual voxelwise clustering results defined a "group graph," with weighted connections between the cortical voxels reflecting the level of consistency of the clustering results over the group of subjects. The voxels in the group graph were selected from the group cortical segmentation map, which resulted from overlapping the individual cortical segmentation maps. The group graph was clustered, clustering voxels into resting-state networks that showed a high level of functional connectivity consistently over the group of subjects. Using the normalized cut group clustering approach, the number of RSN clusters are defined as an optimal clustering fit of the group graph, which is defined as a clustering fit that minimizes the total cost of partitioning the graph into separate networks (van den Heuvel et al., 2008). This optimization procedure resulted in an optimal clustering fit of the data in seven RSNs (Fig. 1). Group clustering revealed the extensively described default mode network (Raichle et al., 2001; Greicius et al., 2003), overlapping the PCC [Brodmann area (BA) 23/31], bilateral middle/superior temporal gyrus (BA 21/39) and inferior/superior parietal cortex (SPC) (BA 39/40), and frontal cortices, including both superior frontal cortex (BA 8/9) and MFC (BA 10/11). The other clustered RSNs included two lateralized frontoparietal networks, a motor/sensory/auditory network, a network consisting of insula and anterior cingulate cortex, and two singular networks that overlapped the medial frontal gyrus and a posterior part of BA 7 (Fig. 1). The clustered RSNs showed resemblance with the resting-state networks found by previous studies reporting on the groupwise selection of resting-state networks (Beckmann et al., 2005; Damoiseaux et al., 2006; De Luca et al., 2006; van den Heuvel et al., 2008).

Functional connectivity. The PCC and MFC were selected from the default mode network cluster map (Fig. 1a). For each of the individual datasets, the functional connectivity between the PCC and MFC was calculated as follows. The fMRI time series of all voxels were filtered using a bandpass filter, extracting the low resting-state frequencies of interest (0.01-0.08 Hz) (Biswal et al., 1995, 1997; Cordes et al., 2001; Achard et al., 2006). The representative time series of the PCC and MFC were obtained by averaging the time series of the voxels within these regions. Next, the level of functional connectivity between the PCC and MFC regions was computed as a partial correlation between their filtered time series, controlling for third-party influences of other RSN regions (Stam, 2004; Sun et al., 2004; Salvador et al., 2005; Achard et al., 2006; Liu et al., 2008). Besides the default mode network, the group clustering revealed six other resting-state networks, which in total consisted of 13 anatomically separate regions. For each of these 13 other RSN regions and the two bilateral SPC regions of the default mode network, representative time series were computed by averaging the time series of all voxels within this particular RSN region (Liu et al., 2008). The partial correlation between the time series of the PCC and MFC was computed by correlating the resting-state time series of the PCC and MFC, factoring out the contributions of the time series of the other $13 \mathrm{RSN}$ regions and the two bilateral SPC regions of the default mode network (Fig. 1a). This procedure was similar to the methodology used in previous studies examining functional connectivity between brain regions (Salvador et al., 2005; Achard et al., 2006; Liu et al., 2008). In these studies, the brain was partitioned into a fixed number of regions based on a predefined anatomical template, normally $\sim 90$ regions, and partial correlations between the time 


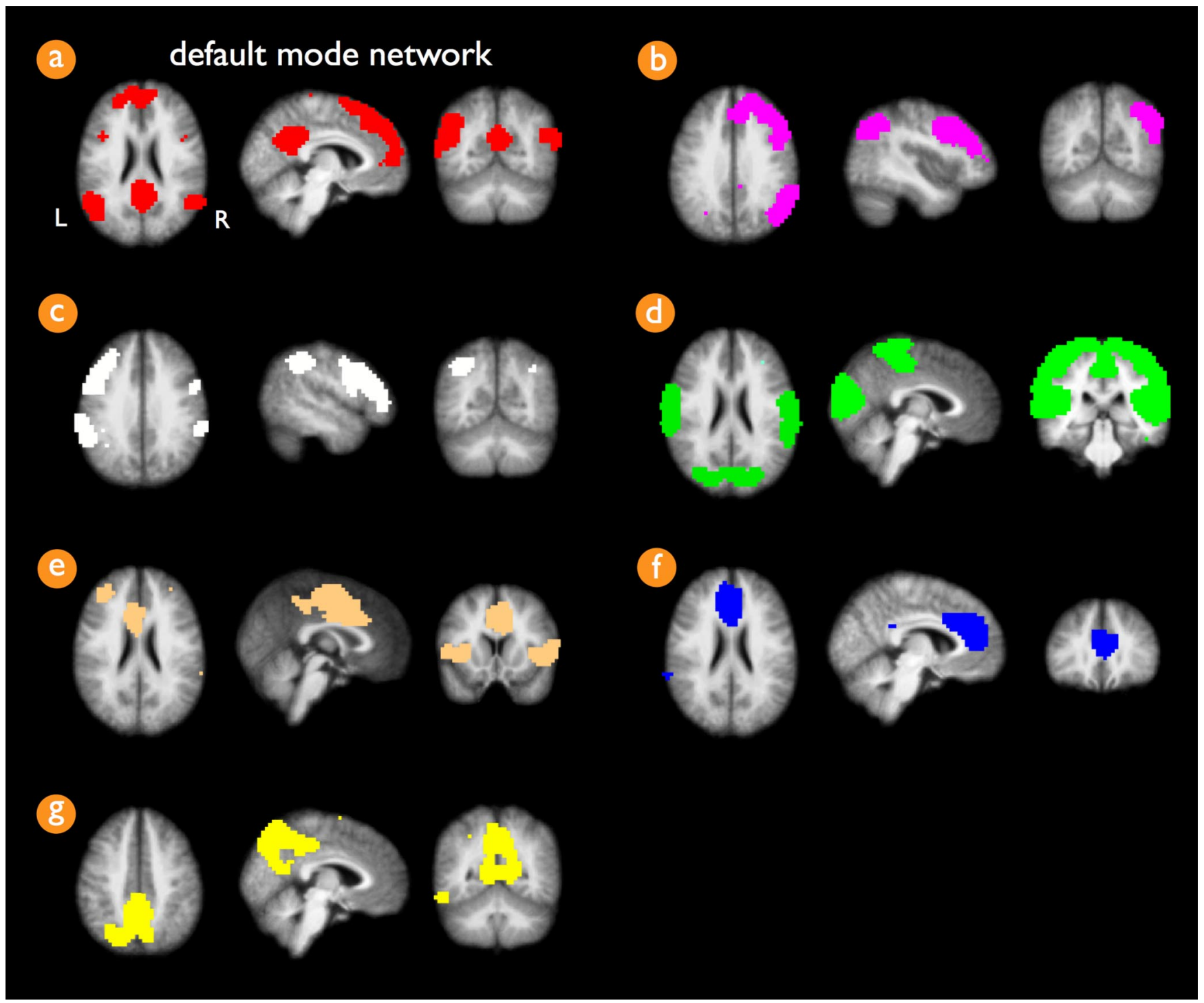

Figure 1. Group clustered resting-state networks. Normalized cut group clustering of the resting-state time series of the group of 45 subjects revealed seven resting-state networks. Cluster a (a) shows the default mode network, consisting of frontal regions, including superior frontal gyrus (BA 8/9) and medial frontal gyrus (BA 10/11) and precuneus/posterior cingulate cortex (BA 23/31) and bilateral regions overlapping middle/superior temporal tyrus (BA 21/39) and inferior/superior parietal cortex (BA 39/40). Clusters b and c (b, $c$ ) show lateralized frontoparietal networks in the right and left hemisphere, overlapping regions in the superior parietal lobule, inferior parietal lobule, supramarginal gyrus (BA 40), and medial and superior frontal gyrus (BA 8/9). Cluster d (d) shows a combined network of both visual and motor regions, consisting of medial, lateral, and superior occipital gyrus and peristriate regions (BA 17/18/19), precentral (BA4), and postcentral gyrus (BA3/1/2). Cluster e (e) shows a network of cingulate gyrus (BA 24) and bilateral insular and superior temporal gyrus (BA 13/22), a network that is also commonly found in resting-state studies. Cluster $f(f)$ involves a singular region consisting of a medial part of the medial frontal gyrus (BA 9) and cingulate gyrus (BA 32). Cluster $g(\boldsymbol{g})$ involves a singular region consisting of a posterior part of BA 7. L, Left; R, right.

series of each pair of regions was computed by factoring out the contributions of the time series of the other 88 regions. In our study, the third-party regions were defined as the brain regions of the other RSNs (Fig. 1) that resulted from the group clustering. The use of a "partial correlation" ensured the examination of the specific level of functional connectivity between the PCC and MFC (Salvador et al., 2005; Achard et al., 2006; Liu et al., 2008). To verify this specificity, the "straight correlation" coefficient (i.e., nonpartialized correlation) between the filtered time series of the PCC and MFC was computed, correlating the PMRI time series of the PCC and MFC, without factoring out the contributions of the other RSN regions. In addition, to examine the level of overall default mode functional connectivity, the time series of the PCC and MFC regions were correlated, factoring out the third-party effects of the other RSN regions (Fig. $1 b-g$ ) but not the effects of the two SPC regions of the default mode network (Fig. 1). Finally, a Fisher's $r$-to- $z$ transformation was used to improve the normality of the partial correlation coefficients (Salvador et al., 2005; Fox and Raichle, 2007). The partial correlation Fisher's $z$ coefficient reflected the "unique" level of functional connectivity between the PCC and MFC of the default mode network (Salvador et al., 2005; Achard et al., 2006).

Selection of interconnecting tracts. It was verified for each individual dataset whether or not the two PCC and MFC regions of interest were interconnected with white matter tracts. First, to overlap the regions of interest with the individual structural DTI data, the default mode network cluster map was registered to the individual unweighted B0 image (using the reversed normalization and registration parameters generated in the matching of $\mathrm{B} 0$ and $\mathrm{T} 1$ images). Next, from the total collection of reconstructed tracts, the tracts that touched both the PCC and the MFC were selected, using a three step procedure (Fig. 2). From the total collection of reconstructed tracts (Fig. 2, step 1), the tracts that touched the PCC were selected (Fig. 2, step 2). Next, from the resulting tracts, the tracts that touched the MFC were selected (Fig. 2, step 3). To enable group comparison, the resulting tracts were normalized using the registration and normalization parameters of the $\mathrm{B} 0$ image. The mean FA value of the resulting tracts, an estimate of the microstructural organization within these white matter tracts (Beaulieu, 2002), was calculated by 


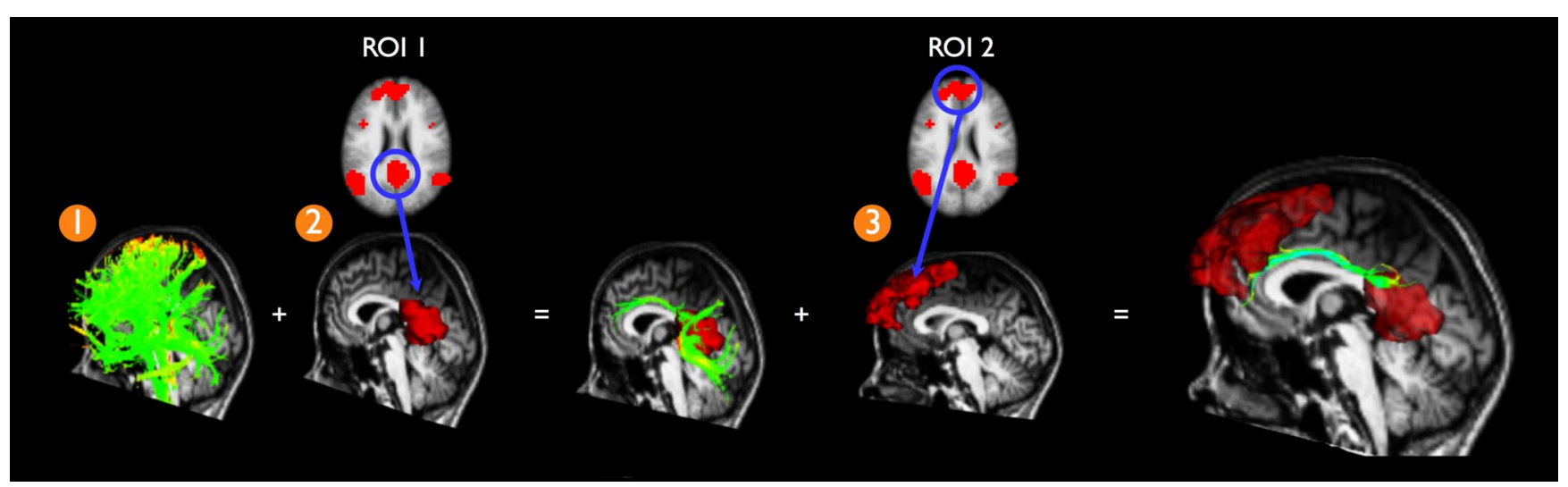

Figure 2. Selection of tracts that interconnect the regions of the default mode network. In each individual dataset, interconnecting tracts between the PCC and MFC of the default mode network were selected as follows. First, the FACT algorithm was used to trace the total collection of tracts in the human brain. Twenty-seven fiber seeds were started in all voxels of the brain, reconstructing the total collection of fibers (step 1). Second, the PCC was selected from the default mode network cluster map (Fig. 1a). Fibers that touched the PCC region were selected (step 2). Third, the MFC was selected from the default mode network cluster map and from the resulting fibers of step 2 the fibers that touched the MFC regions were selected (step 3). This procedure resulted in the fibers that touched both regions of interest (ROI), selecting the fibers that interconnected the PCC and MFC of the default mode network.

averaging the FA values of the points along the selected left and right hemispheric tracts. This procedure was repeated for each individual dataset, obtaining a mean FA value for the connecting tracts in each subject. In addition, for each individual DTI dataset, the mean FA value of the total collection of fibers in the brain was computed.

Association between structural and functional connectivity. The association between the level of structural connectivity and the level of functional connectivity of the default mode network was computed by correlating the mean FA value of the connecting tracts with the unique level of functional connectivity (Fisher's $z$ score) between the PCC and MFC over the group of subjects, correcting for age. Possible age effects were regressed out of the FA and functional connectivity measures separately, because recent studies have demonstrated that normal aging is associated with both decreased microstructural organization (i.e., lower FA values) of the cingulum tract (Andrews-Hanna et al., 2007) as well as decreased levels of default mode functional connectivity (Andrews-Hanna et al., 2007; Damoiseaux et al., 2008). In addition, an alternative approach to correct for aging effects was examined by taking age as a covariate in assessing the correlation between default mode functional connectivity and mean cingulum FA values. Furthermore, to examine whether the association between mean FA and default mode functional connectivity was specific to the interconnecting cingulum tracts or instead was related to a more global effect, two additional analyses were performed. One, the level of functional connectivity between the PCC and MFC regions was correlated with the mean FA value of the total collection of reconstructed fibers in the brain. Two, the functional connectivity levels of the other clustered RSNs were correlated with the mean FA value of the cingulum tract. Group clustering revealed six additional RSNs, with four RSNs consisting of two or more anatomically separate cortical regions (Fig. 1). In total, these four RSNs consisted of 11 regions. Functional connectivity measures of these RSNs were computed in a similar manner as the computation of the level of functional connectivity of the default mode network. The level of functional connectivity was defined as the partial correlation between the filtered resting-state time series of the regions of the selected RSN, correcting for third-party effects of the time series of the regions of the other clustered RSNs. The resulting partial correlation coefficient was correlated with the mean FA value of the cingulum, after controlling for age on both measures.

\section{Results}

Normalized cut group clustering of the resting-state fMRI data revealed seven resting-state networks, including the often reported default mode network (Fig. 1a). The level of overall default mode connectivity (i.e., the correlation between the PCC and MFC, factoring out the effects of the other RSNs but not the two SPC regions) was significantly non-zero over the group of subjects (mean $\pm \mathrm{SD}, 0.51 \pm 0.23 ; p<0.001$ ). The level of unique default mode functional connectivity between the PCC and MFC was found to be significantly non-zero over the group of subjects (mean $\pm \mathrm{SD}, 0.19 \pm 0.24 ; p<0.001)$. All 45 subjects showed interconnecting tracts between the PCC and MFC of the default mode network. The normalized connecting tracts of all 45 subjects are shown in Figure 3, together with the PCC and MFC regions of interest from the default mode network cluster map. The selected connecting tracts reflected the left and right cingulum fiber bundle (Wakana et al., 2004; Lawes et al., 2008). The average \pm SD FA value of the cingulum tracts over the group of subjects was $0.52 \pm 0.05$. Correlating the straight correlation coefficient (i.e., nonpartialized) between the PCC and MFC time series with the mean FA value of the cingulum tracts revealed a positive but nonsignificant association $(r=0.18 ; p=0.17$; mean $\pm \mathrm{SD}, 0.18 \pm 0.33$, corrected for age). Correlating the level of overall default mode functional connectivity with the FA value of the cingulum tract revealed a positive trend $(r=0.23 ; p=0.07$, corrected for age). Correlating the partial correlation coefficient as a measure of the unique level of default mode PCC-MFC functional connectivity with the mean FA value of the cingulum tracts revealed a significant positive association $(r=0.29$; $p<$ 0.05 ) (Fig. 4), after controlling for age on each measure. The association between default mode functional connectivity and mean cingulum FA values was also examined by taking age as a covariate when assessing the correlation of mean cingulum FA and resting-state functional connectivity measures. This alternative approach also revealed a significant association between default mode functional connectivity and mean cingulum FA ( $r=$ $0.34 ; p<0.05)$. Furthermore, the association between the level of default mode functional connectivity and FA measures was found to be specific to the cingulum because no significant association was found between the level of default mode functional connectivity and the mean FA value of the total collection of reconstructed tracts in the brain (i.e., all other white matter tracts in the brain) $(r=0.12 ; p=0.37)$. In addition, no significant correlation was found between the mean FA value of the cingulum tract and the level of functional connectivity between the regions of the other RSNs (RSN b, Fig. $1 b: r=-0.16, p=0.29$; RSN c, Fig. $1 c: r=-0.11, p=0.43$; RSN d, Fig. 1d, left-right primary motor/sensory cortex: $r=0.05, p=0.74$; RSN d, Fig. $1 d$, left-right primary visual cortex: $r=0.16, p=0.33$; RSN e, Fig. $1 e$, 


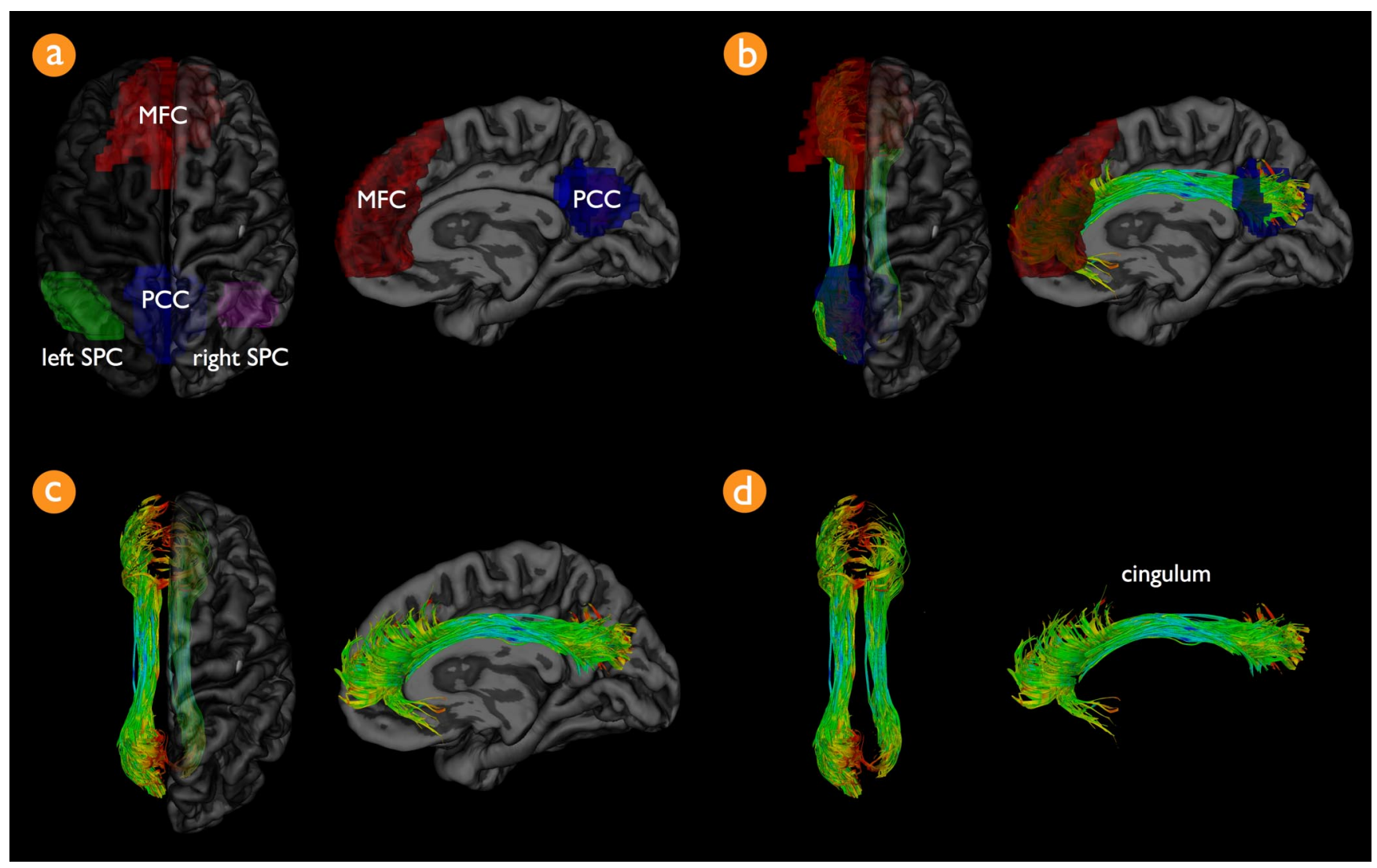

Figure 3. The cingulum tract connects precuneus and posterior cingulate cortex and medial frontal cortex of the default mode network. $\boldsymbol{a}$ shows the regions of interest of the default mode network on a 3D rendering of the group-averaged normalized T1 scan. The default mode network was selected by using a normalized cut group clustering approach of the resting-state data. Clustering revealed the default mode network, including the PCC (blue region of interest), MFC (red region of interest), and bilateral SPC (green and magenta regions). $\boldsymbol{b}$ - $\boldsymbol{d}$ show the interconnecting tracts between the PCC and MFC regions over all subjects combined. $\boldsymbol{b}$ shows both the tracts together with the PCC (blue) and MFC (red) regions of the default mode network.

left-right inferior/superior temporal cortex: $r=0.08, p=0.61$; RSN e, Fig. 1e, left inferior/superior temporal cortex-anterior cingulate gyrus: $r=0.03, p=0.85$; RSN e, Fig. $1 e$ : right inferior/ superior temporal cortex-anterior cingulate gyrus: $r=0.17, p=$ $0.26)$. No significant association was found between mean cingulum FA values and age $(r=-0.05 ; p=0.73)$ or the level of default mode functional connectivity and age $(r=0.06 ; p=0.68)$, which is likely to result from the relative young age of the subjects that were included in this study (mean \pm SD age, $24.8 \pm 4.8$ years).

\section{Discussion}

The main finding of this study is that the microstructural organization (fractional anisotropy) of the interconnecting cingulum tract was found to be directly associated with the level of functional connectivity of the default mode network. We combined resting-state functional MRI and structural DTI in a group of 45 subjects on a 3 tesla MR scanner. The cingulum tract was confirmed to interconnect the PCC and MFC of the default mode network in all subjects (Fig. 3). Furthermore, the mean FA value of the cingulum tract was positively correlated with the level of unique coherency between the resting-state fMRI time series of the PCC and MFC of the default mode network (Fig. 4). These results suggest a specific association between the microstructural organization of the cingulum tract and the level of default mode functional connectivity. Our findings suggest an important role of the cingulum tract in the default mode network.

The observed interconnecting role of the cingulum tract in the default mode network is consistent with studies demonstrating that the cingulum tract forms a direct neuroanatomical link between the precuneus and medial frontal cortex (Wakana et al., 2004; Schmahmann et al., 2007; Lawes et al., 2008). Our results are coherent with the recent study of Greicius et al. (2008) reporting on an important role of the cingulum tract in interconnecting PCC and MFC of the default mode network. This direct anatomical connection reflects a vast number of axonal connections between the PCC and MFC, responsible for the facilitation of neuronal communication between these regions. This communication is likely to be ongoing during rest as suggested by the high level of neuronal activity (Gusnard et al., 2001; Raichle et al., 2001; Raichle and Snyder, 2007; Damoiseaux et al., 2008) and the observed high level of resting-state synchronization between the PCC and MFC (Greicius et al., 2003; Damoiseaux et al., 2008). Furthermore, our results show that the temporal coherency between the activation patterns of the PCC and MFC regions is specifically related to the microstructural organization (i.e., FA value) of the cingulum. It is believed that the major contribution to the directional-dependent diffusion signal is attributable to axonal membranes hindering the diffusion process of water molecules (Beaulieu, 2002), and increased FA values may be associated with a more dense packing of axonal fibers. In this context, it is reasonable to speculate about a positive relationship between the microstructural organization of white matter tracts, reflected by the FA value, and the level of default mode neuronal communication. A possible interpretation of our results could be that a higher level of microstructural organization of the cingulum tract 

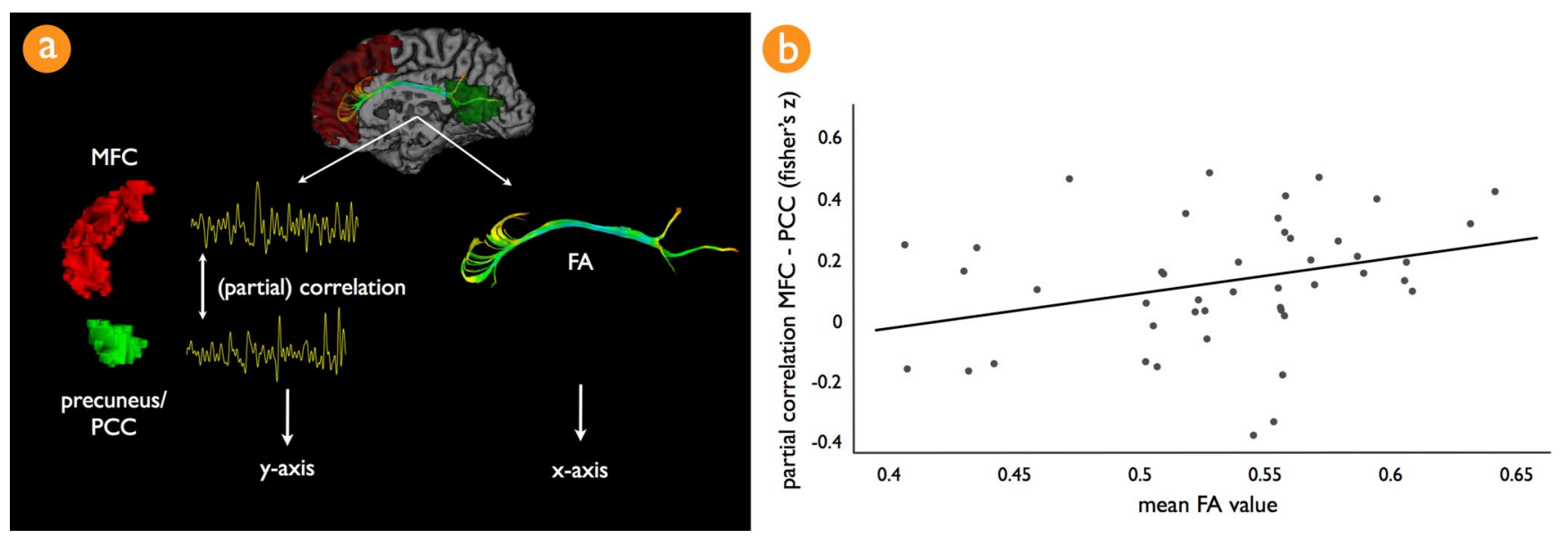

Figure 4. Association between the level of default mode functional connectivity and the microstructural organization of the cingulum tract. $\boldsymbol{a}$ illustrates the analysis that was used to compute the level of functional and structural connectivity between the regions of interest from the default mode network. In each individual dataset, the spatially averaged filtered rest-recorded time series $(0.01-0.08 \mathrm{~Hz})$ of the PCC and MFC of the default mode network cluster map were correlated, correcting for third-party effects. This resulted in a partial correlation between the PCC and MFC regions, which reflected the unique level of functional connectivity between these two key regions of the default mode network. The normality of the partial correlation coefficient was improved by using a Fisher's $r$-to-z transformation. For each individual dataset, the mean FA value of the found interconnecting cingulum tract was calculated by averaging the $F A$ values along all points of the found interconnecting tracts. $\boldsymbol{b}$, Correlating the level of default mode functional connectivity and mean cingulum FA revealed a significant positive correlation, correcting for age $(r=0.29 ; p<0.05)$. This suggests a direct positive association between the microstructural organization of the cingulum tract and the level of functional connectivity of the default mode network during rest. Note that the partial correlation between the PCC and MFC regions represented the partial correlation between these regions, factoring out the pairwise correlations between the time series of the other resting-state network regions, including the two lateralized superior parietal regions of the default mode network. This relationship was found to be specific for the cingulum tract, because no significant association was found between the partial correlation between the PCC and MFC and the mean FA value of the total collection of reconstructed tracts in the brain. Furthermore, functional connectivity measures between the regions of the other clustered resting-state networks (Fig. 1, networks b-g) did not show a significant association with the mean FA value of the cingulum tract.

is associated with increased communication between PCC and MFC of the default mode network during rest.

Supporting evidence for our findings comes from studies reporting on decreased default mode functional connectivity in combination with studies reporting on degenerative brain abnormalities in the cingulum bundle. Alzheimer's disease patients show a decreased level of functional connectivity within the default mode network (Greicius et al., 2004; Rombouts et al., 2005) as well as reduced FA values in the cingulum tract (Xie et al., 2005; Zhang et al., 2007). In addition, normal aging has been associated with decreased resting-state activation within the default mode network (Andrews-Hanna et al., 2007; Damoiseaux et al., 2008) and with lower FA values of the cingulum (Makris et al., 2007; Schneiderman et al., 2007; Yoon et al., 2008). Furthermore, decreased default mode functional connectivity (Friston, 1999; Liang et al., 2006; Micheloyannis et al., 2006; Bluhm et al., 2007; Salvador et al., 2007; Williamson, 2007; Liu et al., 2008) and reduced microstructural organization of the cingulum tract (Sun et al., 2003; Nestor et al., 2007) have been suggested to play an important role in schizophrenia.

The results of this study can be interpreted as supporting evidence for a general association between structural and functional connectivity in the human brain (Koch et al. 2002; Toosy et al., 2004; Greicius et al., 2008). A number of recent studies have suggested a direct link between white matter organization and fMRI BOLD activation patterns. FA values of splenial fibers have been associated with BOLD activation in the primary visual regions (Toosy et al., 2004) and interhemispheric synchronization in the developing brain (Fornari et al., 2007). Furthermore, complete section of the corpus callosum has been reported to result in loss of interhemispheric resting-state functional connectivity (Johnston et al., 2008).

What does it mean when subjects show a higher level of resting-state default mode neuronal activation and synchronization? The results of our study cannot be used to draw conclusions about the functional relevance of the default mode network. However, other studies have suggested that increased recruitment of the default mode network is related to the tendency of a person's mind to wander (Mason et al., 2007), and it may be linked to the integration of cognitive and emotional processing (Greicius et al., 2003) and relating oneself to the outside world (Gusnard et al., 2001). These processes are likely to be ongoing during rest and to involve the integration of multiple cognitive functions. Structural pathways interconnecting the regions of default mode network may facilitate this kind of functional integration. It is of interest to further examine the found structuralfunctional relationship and its effect on cognitive and emotional processes that are related to default mode activation (AndrewsHanna et al., 2007).

The level of overall default mode functional connectivity was significantly non-zero over the group of 45 subjects (Greicius et al., 2003; Beckmann et al., 2005; Salvador et al., 2005; De Luca et al., 2006; Damoiseaux et al., 2008; van den Heuvel et al., 2008). Interestingly, the unique level of default mode functional connectivity between the PCC and MFC (i.e., controlling for the third-party influences of the two SPC regions) was found to be negative in 10 of the 45 subjects, with two subjects showing a partial correlation lower than -0.2 (Fig. $4 b$ ). Negative levels of default mode connectivity have been related to advanced aging (Andrews-Hanna et al., 2007). However, such an effect could not be concluded from our results. The 10 subjects who showed a negative level of connectivity were not significantly older than the 35 subjects who showed a positive level of default mode connectivity. This is likely to result from the relatively young age of the included group of subjects (mean age, 24.8 years). Most other resting-state studies have reported only positive levels of default mode connectivity in young adults (Greicius et al., 2003). However, these studies have mainly focused on the level of overall default mode connectivity, defined as a straight correlation between the resting-state time series of the PCC and MFC. In our 
study, we focused on the level of unique default mode connectivity between the PCC and MFC (by factoring out the contribution of the two SPC regions), because these are the two regions of the default mode network that are specifically connected by the cingulum. Indeed, supporting the results of previous resting-state studies, the level of overall default mode connectivity was found to be positive in all 45 subjects (mean, 0.51). Future studies are needed to examine the meaning of these specific negative synchronization patterns and how they relate to cognitive processes that involve default mode network activity. In this study, negative synchronization patterns between the PCC and MFC were interpreted as lower levels of default mode functional connectivity.

Some limitations of this study have to be taken into account when interpreting its results. The PCC and MFC regions are the most often reported regions of the default mode network. Therefore, the focus of this study was on the tracts that interconnected these two regions of the default mode network. As mentioned, bilateral parietal and temporal cortices are also found to participate in the default mode network (Raichle et al., 2001; Greicius et al., 2003; Fox et al., 2005; Damoiseaux et al., 2006) (Fig. 3). However, tracts connecting these regions are likely to cross other white matter pathways, making the reconstruction of these tracts more difficult. Future studies are aimed to examine these tracts using other methods of representing the diffusion signal in combination with other tractography algorithms. Furthermore, the level of functional connectivity is expressed as a (partial) correlation between the rest-recorded BOLD time series, believed to result from synchronization of neuronal activation (Biswal et al., 1997; Cordes et al., 2001; Greicius et al., 2003; Salvador et al., 2005; Buckner and Vincent, 2007). However, it has been suggested that physiological temporal patterns, including respiratory and cardiac oscillations, could confound the BOLD signal (Wise et al., 2004), making the resting-state correlations less specific. We used a high temporal fMRI acquisition sequence to minimize these effects (Cordes et al., 2001).

In this study, we found a significant role for the cingulum tract in the default mode network in interconnecting the precuneus/ posterior cingulate cortex and medial frontal cortex, key regions of the default mode network. Higher fractional anisotropy values of the cingulum were found to be associated with increased default mode functional connectivity, suggesting a direct association between the microstructural organization of the cingulum tract and the level of neuronal synchronization between key regions of the default mode network. Our results suggest an important role for the cingulum tract in default mode functional connectivity.

\section{References}

Achard S, Salvador R, Whitcher B, Suckling J, Bullmore E (2006) A resilient, low-frequency, small-world human brain functional network with highly connected association cortical hubs. J Neurosci 26:63-72.

Andersson JL, Skare S (2002) A model-based method for retrospective correction of geometric distortions in diffusion-weighted EPI. Neuroimage 16:177-199.

Andersson JL, Skare S, Ashburner J (2003) How to correct susceptibility distortions in spin-echo echo-planar images: application to diffusion tensor imaging. Neuroimage 20:870-888.

Andrews-Hanna JR, Snyder AZ, Vincent JL, Lustig C, Head D, Raichle ME, Buckner RL (2007) Disruption of large-scale brain systems in advanced aging. Neuron 56:924-935.

Beaulieu C (2002) The basis of anisotropic water diffusion in the nervous system: a technical review. NMR Biomed 15:435-455.

Beckmann CF, DeLuca M, Devlin JT, Smith SM (2005) Investigations into resting-state connectivity using independent component analysis. Philos Trans R Soc Lond B Biol Sci 360:1001-1013.
Biswal B, Yetkin FZ, Haughton VM, Hyde JS (1995) Functional connectivity in the motor cortex of resting human brain using echo-planar MRI. Magn Reson Med 34:537-541.

Biswal BB, Van Kylen J, Hyde JS (1997) Simultaneous assessment of flow and BOLD signals in resting-state functional connectivity maps. NMR Biomed 10:165-170.

Bluhm RL, Miller J, Lanius RA, Osuch EA, Boksman K, Neufeld RW, Théberge J, Schaefer B, Williamson P (2007) Spontaneous low-frequency fluctuations in the BOLD signal in schizophrenic patients: anomalies in the default network. Schizophr Bull 33:1004-1012.

Buckner RL, Vincent JL (2007) Unrest at rest: default activity and spontaneous network correlations. Neuroimage 37:1091-1096; discussion 1097-1099.

Chang LC, Jones DK, Pierpaoli C (2005) RESTORE: robust estimation of tensors by outlier rejection. Magn Reson Med 53:1088-1095.

Collins DL, Neelin P, Peters TM, Evans AC (1994) Automatic 3D intersubject registration of MR volumetric data in standardized Talairach space. J Comput Assist Tomogr 18:192-205.

Corbetta M, Kincade JM, Ollinger JM, McAvoy MP, Shulman GL (2000) Voluntary orienting is dissociated from target detection in human posterior parietal cortex. Nat Neurosci 3:292-297.

Cordes D, Haughton VM, Arfanakis K, Carew JD, Turski PA, Moritz CH, Quigley MA, Meyerand ME (2001) Frequencies contributing to functional connectivity in the cerebral cortex in "resting-state" data. AJNR Am J Neuroradiol 22:1326-1333.

Damoiseaux JS, Rombouts SA, Barkhof F, Scheltens P, Stam CJ, Smith SM, Beckmann CF (2006) Consistent resting-state networks across healthy subjects. Proc Natl Acad Sci U S A 103:13848-13853.

Damoiseaux JS, Beckmann CF, Arigita EJ, Barkhof F, Scheltens P, Stam CJ, Smith SM, Rombouts SA (2008) Reduced resting-state brain activity in the "default network" in normal aging. Cereb Cortex 18:1856-1864.

De Luca M, Beckmann CF, De Stefano N, Matthews PM, Smith SM (2006) fMRI resting state networks define distinct modes of long-distance interactions in the human brain. Neuroimage 29:1359-1367.

Fornari E, Knyazeva MG, Meuli R, Maeder P (2007) Myelination shapes functional activity in the developing brain. Neuroimage 38:511-518.

Fox MD, Raichle ME (2007) Spontaneous fluctuations in brain activity observed with functional magnetic resonance imaging. Nat Rev Neurosci 8:700-711.

Fox MD, Snyder AZ, Vincent JL, Corbetta M, Van Essen DC, Raichle ME (2005) The human brain is intrinsically organized into dynamic, anticorrelated functional networks. Proc Natl Acad Sci U S A 102:9673-9678.

Friston KJ (1999) Schizophrenia and the disconnection hypothesis. Acta Psychiatr Scand Suppl 395:68-79.

Golay X, Pruessmann KP, Weiger M, Crelier GR, Folkers PJ, Kollias SS, Boesiger P (2000) PRESTO-SENSE: an ultrafast whole-brain fMRI technique. Magn Reson Med 43:779-786.

Greicius MD, Krasnow B, Reiss AL, Menon V (2003) Functional connectivity in the resting brain: a network analysis of the default mode hypothesis. Proc Natl Acad Sci U S A 100:253-258.

Greicius MD, Srivastava G, Reiss AL, Menon V (2004) Default-mode network activity distinguishes Alzheimer's disease from healthy aging: evidence from functional MRI. Proc Natl Acad Sci U S A 101:4637-4642.

Greicius MD, Supekar K, Menon V, Dougherty RF (2008) Resting-state functional connectivity reflects structural connectivity in the default mode network. Cereb Cortex, in press.

Gusnard DA, Raichle ME, Raichle ME (2001) Searching for a baseline: functional imaging and the resting human brain. Nat Rev Neurosci 2:685-694.

Johnston JM, Vaishnavi SN, Smyth MD, Zhang D, He BJ, Zempel JM, Shimony JS, Snyder AZ, Raichle ME (2008) Loss of resting interhemispheric functional connectivity after complete section of the corpus callosum. J Neurosci 28:6453-6458.

Jones DK (2004) The effect of gradient sampling schemes on measures derived from diffusion tensor MRI: a Monte Carlo study. Magn Reson Med 51:807-815.

Jones DK, Horsfield MA, Simmons A (1999) Optimal strategies for measuring diffusion in anisotropic systems by magnetic resonance imaging. Magn Reson Med 42:515-525.

Kim JH, Loy DN, Liang HF, Trinkaus K, Schmidt RE, Song SK (2007) Noninvasive diffusion tensor imaging of evolving white matter pathology in a mouse model of acute spinal cord injury. Magn Reson Med 58:253-260. 
Koch MA, Norris DG, Hund-Georgiadis M (2002) An investigation of functional and anatomical connectivity using magnetic resonance imaging. Neuroimage 16:241-250.

Lawes IN, Barrick TR, Murugam V, Spierings N, Evans DR, Song M, Clark CA (2008) Atlas-based segmentation of white matter tracts of the human brain using diffusion tensor tractography and comparison with classical dissection. Neuroimage 39:62-79.

Liang M, Zhou Y, Jiang T, Liu Z, Tian L, Liu H, Hao Y (2006) Widespread functional disconnectivity in schizophrenia with resting-state functional magnetic resonance imaging. Neuroreport 17:209-213.

Liu Y, Liang M, Zhou Y, He Y, Hao Y, Song M, Yu C, Liu H, Liu Z, Jiang T (2008) Disrupted small-world networks in schizophrenia. Brain 131:945-961.

Makris N, Papadimitriou GM, van der Kouwe A, Kennedy DN, Hodge SM, Dale AM, Benner T, Wald LL, Wu O, Tuch DS, Caviness VS, Moore TL, Killiany RJ, Moss MB, Rosene DL (2007) Frontal connections and cognitive changes in normal aging rhesus monkeys: a DTI study. Neurobiol Aging 28:1556-1567.

Mason MF, Norton MI, Van Horn JD, Wegner DM, Grafton ST, Macrae CN (2007) Wandering minds: the default network and stimulusindependent thought. Science 315:393-395.

Micheloyannis S, Pachou E, Stam CJ, Breakspear M, Bitsios P, Vourkas M, Erimaki S, Zervakis M (2006) Small-world networks and disturbed functional connectivity in schizophrenia. Schizophr Res 87:60-66.

Mori S, van Zijl PC (2002) Fiber tracking: principles and strategies-a technical review. NMR Biomed 15:468-480.

Mori S, Crain BJ, Chacko VP, van Zijl PC (1999) Three-dimensional tracking of axonal projections in the brain by magnetic resonance imaging. Ann Neurol 45:265-269.

Neggers SF, Hermans EJ, Ramsey NF (2008) Enhanced sensitivity with fast three-dimensional blood-oxygen-level-dependent functional MRI: comparison of SENSE-PRESTO and 2D-EPI at $3 \mathrm{~T}$. NMR Biomed 21:663-676.

Nestor PG, Kubicki M, Spencer KM, Niznikiewicz M, McCarley RW, Shenton ME (2007) Attentional networks and cingulum bundle in chronic schizophrenia. Schizophr Res 90:308-315.

Northoff G, Bermpohl F (2004) Cortical midline structures and the self. Trends Cogn Sci 8:102-107.

Northoff G, Heinzel A, de Greck M, Bermpohl F, Dobrowolny H, Panksepp J (2006) Self-referential processing in our brain-a meta-analysis of imaging studies on the self. Neuroimage 31:440-457.

Raichle ME, Snyder AZ (2007) A default mode of brain function: A brief history of an evolving idea. Neuroimage 37:1083-1090; discussion 1097-1099.

Raichle ME, MacLeod AM, Snyder AZ, Powers WJ, Gusnard DA, Shulman GL (2001) A default mode of brain function. Proc Natl Acad Sci U S A 98:676-682.

Rombouts SA, Barkhof F, Goekoop R, Stam CJ, Scheltens P (2005) Altered resting state networks in mild cognitive impairment and mild Alzheimer's disease: an fMRI study. Hum Brain Mapp 26:231-239.

Salvador R, Suckling J, Coleman MR, Pickard JD, Menon D, Bullmore E
(2005) Neurophysiological architecture of functional magnetic resonance images of human brain. Cereb Cortex 15:1332-1342.

Salvador R, Martínez A, Pomarol-Clotet E, Sarró S, Suckling J, Bullmore E (2007) Frequency based mutual information measures between clusters of brain regions in functional magnetic resonance imaging. Neuroimage 35:83-88

Schmahmann JD, Pandya DN, Wang R, Dai G, D'Arceuil HE, de Crespigny AJ, Wedeen VJ (2007) Association fibre pathways of the brain: parallel observations from diffusion spectrum imaging and autoradiography. Brain 130:630-653.

Schmitz TW, Johnson SC (2007) Relevance to self: A brief review and framework of neural systems underlying appraisal. Neurosci Biobehav Rev 31:585-596.

Schneiderman JS, Buchsbaum MS, Haznedar MM, Hazlett EA, Brickman AM, Shihabuddin L, Brand JG, Torosjan Y, Newmark RE, Tang C, Aronowitz J, Paul-Odouard R, Byne W, Hof PR (2007) Diffusion tensor anisotropy in adolescents and adults. Neuropsychobiology 55:96-111.

Stam CJ (2004) Functional connectivity patterns of human magnetoencephalographic recordings: a "small-world" network? Neurosci Lett 355:25-28.

Sun FT, Miller LM, D’Esposito M (2004) Measuring interregional functional connectivity using coherence and partial coherence analyses of fMRI data. Neuroimage 21:647-658.

Sun Z, Wang F, Cui L, Breeze J, Du X, Wang X, Cong Z, Zhang H, Li B, Hong N, Zhang D (2003) Abnormal anterior cingulum in patients with schizophrenia: a diffusion tensor imaging study. Neuroreport 14:1833-1836.

Toosy AT, Ciccarelli O, Parker GJ, Wheeler-Kingshott CA, Miller DH, Thompson AJ (2004) Characterizing function-structure relationships in the human visual system with functional MRI and diffusion tensor imaging. Neuroimage 21:1452-1463.

van den Heuvel MP, Mandl RC, Kahn RS, Hulshoff Pol HE (2007) Anatomical dependence of resting-state networks. Soc Neurosci Abstr 33:761.8.

van den Heuvel MP, Mandl R, Hulshoff Pol H (2008) Normalized group clustering of resting-state fMRI data. PLoS ONE 3:e2001.

Wakana S, Jiang H, Nagae-Poetscher LM, van Zijl PC, Mori S (2004) Fiber tract-based atlas of human white matter anatomy. Radiology 230:77-87.

Williamson P (2007) Are anticorrelated networks in the brain relevant to schizophrenia? Schizophr Bull 33:994-1003.

Wise RG, Ide K, Poulin MJ, Tracey I (2004) Resting fluctuations in arterial carbon dioxide induce significant low frequency variations in BOLD signal. Neuroimage 21:1652-1664.

Xie S, Xiao JX, Wang YH, Wu HK, Gong GL, Jiang XX (2005) Evaluation of bilateral cingulum with tractography in patients with Alzheimer's disease. Neuroreport 16:1275-1278.

Yoon B, Shim YS, Lee KS, Shon YM, Yang DW (2008) Region-specific changes of cerebral white matter during normal aging: a diffusion-tensor analysis. Arch Gerontol Geriatr 47:129-138.

Zhang Y, Schuff N, Jahng GH, Bayne W, Mori S, Schad L, Mueller S, Du AT, Kramer JH, Yaffe K, Chui H, Jagust WJ, Miller BL, Weiner MW (2007) Diffusion tensor imaging of cingulum fibers in mild cognitive impairment and Alzheimer disease. Neurology 68:13-19. 